
cultures

Les cahiers de l'Acedle

10-1 | 2013

Apprendre les langues autrement

\title{
Les progressions linguistiques et scolaires des collégiens nouvellement arrivés, non ou peu scolarisés antérieurement
}

\section{Catherine Mendonça Dias}

\section{(2) OpenEdition \\ 1 Journals}

Édition électronique

URL : http://journals.openedition.org/rdlc/1540

DOI : $10.4000 /$ rdlc. 1540

ISSN : 1958-5772

Éditeur

ACEDLE

Référence électronique

Catherine Mendonça Dias, « Les progressions linguistiques et scolaires des collégiens nouvellement arrivés, non ou peu scolarisés antérieurement », Recherches en didactique des langues et des cultures [En ligne], 10-1 | 2013, mis en ligne le 07 janvier 2013, consulté le 10 décembre 2020. URL : http:// journals.openedition.org/rdlc/1540; DOI : https://doi.org/10.4000/rdlc.1540

Ce document a été généré automatiquement le 10 décembre 2020.

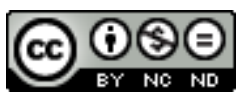

Recherches en didactique des langues et des cultures is licensed under a Creative Commons AttributionNonCommercial-NoDerivatives 4.0 International License 


\title{
Les progressions linguistiques et scolaires des collégiens nouvellement arrivés, non ou peu scolarisés antérieurement
}

\author{
Catherine Mendonça Dias
}

\section{Introduction}

1 Le sigle NSA renvoie aux élèves arrivant en France qui sont "Non Scolarisés Antérieurement". Méconnus, ces élèves n'ont jamais donné lieu à une analyse de leurs parcours et de leurs progrès tandis que nous avons des données pour les élèves scolarisés antérieurement concernant les vitesses d'apprentissage d'une langue seconde (Thomas \& Collier, 2002), les relations avec les compétences initiales, comme en mathématiques (Millon-Fauré, 2011) et les spécificités didactiques et pédagogiques de l'enseignement (Bertrand, Viala \& Vigner, 2000 ; Vigner, 2002 ; Verdelhan-Bourgade, 2002; DavinChnane, 2005).

2 L'accueil des NSA s'avère problématique en raison de leurs compétences scolaires et linguistiques car ils ne maîtrisent pas les pré-requis attendus pour leur âge, ne sont pas familiers de la culture scolaire, ni de l'écrit (Rafoni, 2008) et sont non francophones ou dans une situation de français langue seconde (Cuq, 1991). Leurs vécus sont parfois traumatisants. A leur arrivée en France, ils bénéficient d'un entretien et d'une évaluation, sous la coordination du Casnav (Centre Académique pour la Scolarisation des Nouveaux Arrivants et enfants du Voyage ${ }^{1}$ ). L'analyse des comptes-rendus fait apparaître des facteurs d'hétérogénéité: âge, milieu socioculturel, contexte familial, conditions d'arrivée, biographie langagière, rapport à l'école et compétences à l'écrit et en calcul, données qui nous serviront à pondérer nos résultats ultérieurs et les relations aux conditions de scolarisation. Au collège, certains apprenants rejoignent des dispositifs linguistiques désignés Cla-nsa (Classes d'Accueil pour élèves Non Scolarisés 
Antérieurement) ${ }^{2}$. Pour connaître la spécificité des dispositifs, nous avons soumis un questionnaire numérique à 18 de leurs professeurs.

Notre étude vise à déterminer si les progrès en français - en fonction des profils et des inscriptions éventuelles en Cla-nsa - permettent aux élèves NSA de réussir leur scolarité. Jusqu'alors, les suivis d'élèves primo-arrivants ont été menés dans une démarche sociologique (Schiff, 2003) ou ont concerné un public élargi aux enfants de migrants (Lorcerie, 1995 ; Caille \& Vallet, 1996 ; Chomentowski, 2009). Pourtant, les suivis d'élèves sont recommandés (Ministère de l'Éducation Nationale, 2009: 111; Haut Conseil à l'Intégration, 2010: 21; Abdallah-Pretceille \& Barbot, 2003: 88). Pour notre recherche, nous étudions deux échantillons d'individus. Le premier, qualitatif et étudié dans une démarche prospective, concerne une cohorte de 8 élèves ${ }^{3}$ arrivés dans une même académie au cours de l'année scolaire 2008-2009, mais scolarisés dans différents établissements. Le niveau linguistique atteint au cours des mois est évalué avec les notes obtenues au Delf scolaire (Diplôme d'Études en Langue Française). De plus, lors de leur 3ème année en France ${ }^{4}$, nous leur proposons un test en français qui nous permet de récolter des productions comparables. Le second échantillon, plus quantitatif et analysé suivant un examen rétrospectif, est constitué de 43 élèves qui ont été inscrits dans une même Cla-nsa, entre 2007 et $2011^{5}$. Les progrès en français sont aussi estimés avec les résultats au Delf et leur parcours scolaire est considéré d'après leur orientation et les bulletins scolaires récoltés.

4 Nous présentons nos résultats en trois temps. Dans une première partie, nous décrivons les situations de déscolarisation ou de scolarisation partielle antérieure du public NSA. Notre seconde partie est consacrée à leur scolarisation dans les dispositifs, à leurs enseignants et aux enseignements dispensés. Enfin, notre dernière partie cherche à vérifier les parcours de réussite, à travers les progressions linguistiques et orientations scolaires, au fil du temps et au regard des deux premières parties.

\section{Qui sont les NSA?}

Bien que le sigle NSA renvoie aux élèves "Non Scolarisés Antérieurement", sur le terrain observé, les élèves NSA ont souvent été à l'école antérieurement, mais sur une durée réduite ou dans des conditions inadéquates pour atteindre les compétences scolaires prérequises à leur classe d'âge. L'analyse des comptes-rendus du Casnav nous permet de dresser une typologie en fonction de la scolarisation antérieure, que nous illustrons ciaprès d'exemples rencontrés.

\subsection{De rares élèves n'ont jamais été à l'école}

6 Diego, enfant du voyage, a commencé sa scolarité à 14 ans, sans savoir manipuler un stylo. Lydia, originaire de Luanda, n'a jamais été à l'école et, vendue à un mari à l'âge de 12 ans, la question de l'éducation était close. Des situations financières et le rapport de la famille à l'école peuvent expliquer ces parcours. Pour d'autres, l'absence de scolarisation est compensée par une instruction familiale: Hovannes, adolescent qui habitait en Sibérie, a appris à lire et à compter avec ses parents. Dans le premier degré, des enfants viennent de pays où la scolarité est obligatoire plus tard (à partir de 7 ans, par exemple, en Russie) mais ils ne commencent pas l'école en raison du départ pour la France, préparatifs qui prennent parfois plus de temps que prévu. Bien que le sigle NSA renvoie à 
l'absence de scolarité, ces cas de figure sont rares : par exemple, en 5 ans, sur les 73 élèves ayant été inscrits dans une même Cla-nsa à Creil, seulement 5 n'étaient jamais entrés dans une école auparavant.

\subsection{La plupart des élèves a été "un peu" à l'école}

Dans des contextes agités, l'établissement peut avoir été détruit: ainsi, Farid, un adolescent de la région du Darfour, rapportait avoir commencé l'école à 8 ans et stoppé brutalement sa scolarité alors qu'il en avait 11. Des adolescents peuvent être déscolarisés en raison de conflits dans le pays (cas d'enfants soldats), par nécessité économique (travail comme domestique), pour des raisons de santé ou en fonction d'événements familiaux. Rappelons aussi que, dans certains pays, la scolarité obligatoire s'arrête plus tôt qu'en France. Pour tous ces élèves, nous avons des données objectives se mesurant en années de scolarisation. La dernière circulaire (circulaire $\left.n^{\circ} 2002-100,2012\right)$ retient la notion de "peu" scolarisé antérieurement.

\subsection{La troisième catégorie, "mal scolarisée", est confuse}

La caractéristique de "mal scolarisé" est litigieuse: certains jeunes, inscrits à l'école depuis l'âge de la scolarité obligatoire jusqu'à leur arrivée en France, se trouvent dans une situation proche de l'illettrisme et ils rejoignent une Cla-nsa. Par exemple, Mehmoud a commencé à étudier au Pakistan, a poursuivi aux Etats-Unis et est scolarisé maintenant en France. A chaque nouvelle inscription dans un pays, il a dû acquérir la langue de la scolarisation durant une période où il n'avait pas accès - linguistiquement parlant - aux apprentissages. En France, s'il déchiffrait en ourdou et en anglais, il n'était lecteur dans aucune langue. Les conditions de scolarisation peuvent être interrogées comme pour Isra, qui a connu plusieurs configurations linguistiques et scolaires et dernièrement, venait d'un camp de réfugiés en Somalie. D'autres caractéristiques, qui ne sont pas en ellesmêmes déterminantes, apparaissent: l'apprentissage par cœur dans des écoles coraniques, un sureffectif, le manque de manuels et de matériels, peu d'heures de cours et de nombreuses grèves, un manque de professeurs formés, une scolarité épisodique en raison de difficultés financières, un absentéisme lié au rapport culturel qu'entretient la famille avec l'école, des événements traumatisants... Il est alors difficile d'établir si le jeune était "mal" scolarisé ou en difficultés scolaires, dilemme qui perdure (Francequin, $1998: 78)$.

\section{Les propositions pour les NSA}

\subsection{Les dispositifs}

9 Depuis 2002, ces élèves peuvent rejoindre une classe d'accueil spécifique au collège : la Cla-nsa. Cette même année, dispositifs et élèves considérés NSA sont recensés par la Division de l'Évaluation, de la Prospective et de la Performance (Depp) : 
Figure 1 - Évolution du nombre d'élèves NSA en France métropolitaine, en mai (chiffres de la Depp)

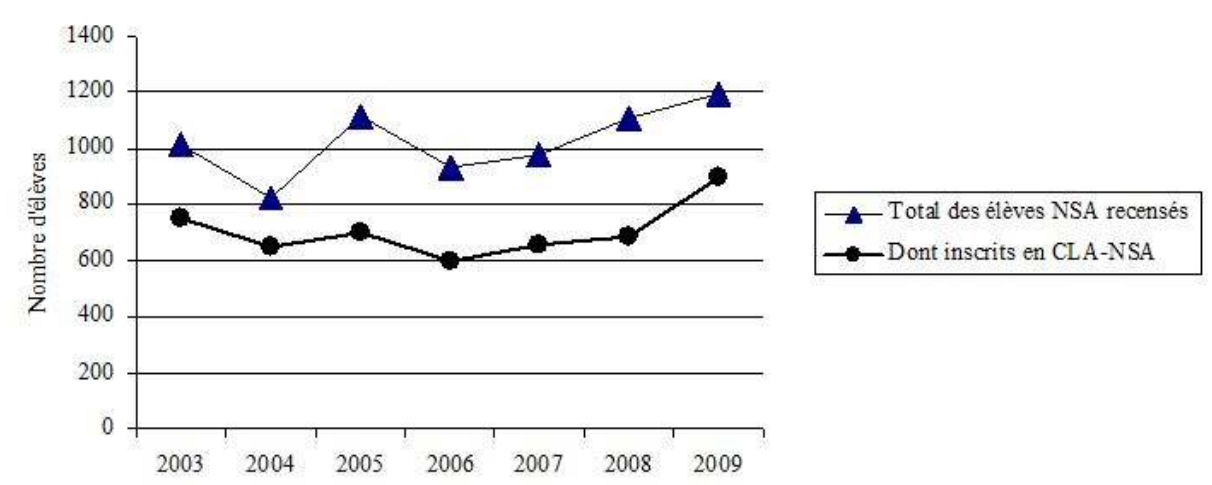

10 En France métropolitaine, l'augmentation constante des élèves s'accompagne du nombre croissant de dispositifs : en mai 2003, on compte 51 classes et en 2009, au moment de notre première enquête, on en compte 64 (certaines peuvent être dans le même établissement) qui scolarisent les trois-quarts des 1193 élèves NSA recensés. La présence des Cla-nsa varie suivant les académies puisque 10 n'en comptent aucune contre 16 académies en comportant généralement une ou deux, voire plus à Paris ou en périphérie (l'académie de Créteil atteint un record avec 16 Cla-nsa). Rafoni rapporte qu'en 2001, "une étude réalisée au Casnav de Versailles nous apprend qu'un élève primo-arrivant sur trois est peu ou pas scolarisé antérieurement" (Rafoni, 2008: 22). Si on se tourne vers les départements d'Outre-mer, ces chiffres augmentent du fait d'un grand nombre d'élèves concernés en Guyane qui dispose de 16 Cla-nsa, en mai 2009.

\subsection{Le cadre institutionnel de l'enseignement en Cla-nsa}

11 En 2009-2010, nous avons observé 18 Cla-nsa sur les 64 recensées. Elles regroupent des jeunes de 12 à 18 ans. Des divergences apparaissent par rapport aux recommandations officielles de 2002 et, par la suite, de 2012. En effet, au vu de l'individualisation nécessaire des activités et de l'ampleur des objectifs, la Cla-nsa ne devrait pas dépasser un effectif de 15 élèves, ce qui n'est pas toujours respecté. Les structures doivent être ouvertes, c'est-àdire que les élèves sont inscrits dans la Cla-nsa et en parallèle, dans une classe du cursus ordinaire où ils devraient suivre au moins les disciplines artistiques et sportives. Pourtant, 7 structures sur les 18 observées organisent les cours de sport en groupe NSA, ce qui révèle les difficultés pour intégrer les élèves et les mettre en contact avec des francophones. Des cours spécifiques dans les autres disciplines devraient être mis en place (des adolescents ne savent pas effectuer une soustraction, etc.) mais là encore, on constate une forte disparité : une structure propose seulement des cours de français tandis que dans une autre, l'ensemble des disciplines est représenté. Les cours spécifiques concernent surtout les mathématiques (16 structures) et l'histoire-géographie (12 structures), pouvant être pris en charge par le professeur de français. Pour 7 enseignants, les emplois du temps sont insatisfaisants en raison du manque d'heures, notamment pour les cours spécifiques. 


\subsection{La formation de 18 professeurs de Cla-nsa}

12 Les enseignants enquêtés, professeurs des écoles ou certifiés en lettres modernes, ont un diplôme en FLE (Français Langue Étrangère) ou connu des expériences de terrain. Ils n'ont pas systématiquement choisi d'exercer en classe d'accueil: il s'est avéré que 6 professeurs ont été sollicités pour enseigner dans ces structures alors qu'ils n'avaient pas bénéficié de formation spécifique sur les élèves allophones. Sept professeurs indiquent avoir besoin de formation sur les techniques d'alphabétisation. Face à des élèves non ou peu lecteurs, ils se retrouvent démunis d'autant plus que les ouvrages sont rares, ce que constatait Rafoni, en introduction de son ouvrage sur l'apprentissage de la lecture en français langue seconde (Rafoni, $2008: 7$ ).

\subsection{L'enseignement en Cla-nsa}

13 En 2001, Jack Lang souhaitait faire progresser l'enseignement aux élèves NSA ${ }^{6}$. Dix ans plus tard, un premier matériel scolaire paraît (Lecoq, 2012). A défaut d'outils spécifiques, les enseignants combinent les méthodes de FLE et celles d'alphabétisation pour enfants ou adultes. Ils introduisent la discipline "français", par la fréquentation de textes littéraires ou l'étude de livres en "français facile". A observer les supports, la Cla-nsa se distingue des classes d'accueil ordinaires par l'alphabétisation. Cependant, que les similarités (manuels de FLE, adaptation de la discipline scolaire "français") ne nous leurrent pas : la vitesse d'apprentissage et la capacité d'abstraction dans les savoirs sont moindres. De plus, face aux besoins des élèves, le professeur de français ne peut pas toujours se soustraire à des exigences d'enseignement pluridisciplinaire qui vise officiellement les compétences de cycle 3, même si les circulaires sont ambiguës quant aux objectifs scolaires réels à atteindre : s'agit-il des compétences de la dyade françaismathématiques, ou bien toutes les disciplines sont-elles concernées ? Enfin, les stratégies d'apprentissage (comment apprendre une leçon, tenir un cahier...) et les méthodologies de travail (remplir un tableau à double entrée, réaliser un contrôle...) représentent aussi une part conséquente du travail en Cla-nsa. Nous allons maintenant nous intéresser aux progrès des élèves.

\section{Les progressions linguistiques et scolaires des élèves}

\subsection{Présentation des 8 élèves de la cohorte}

Notre cohorte compte 8 collégiens catégorisés NSA, âgés de 13 à 15 ans, arrivés au cours de l'année scolaire 2008-2009. Nous en présentons ci-dessous un tableau synthétique : 
Figure 2 - Cohorte des 8 élèves NSA

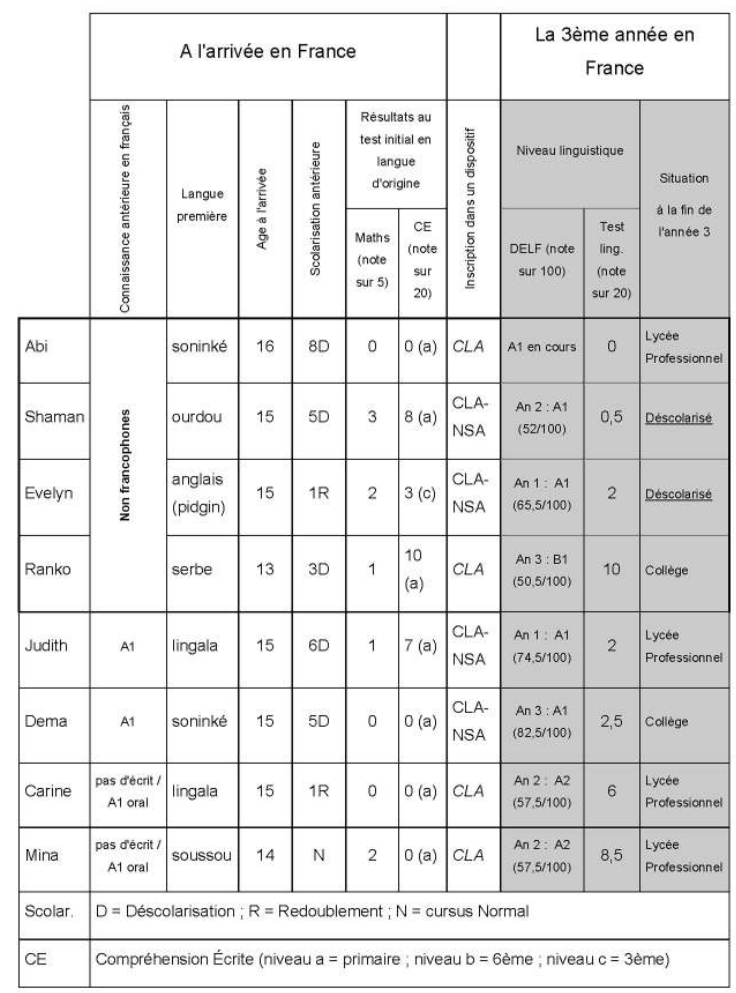

Quatre étaient non francophones et quatre avaient une pratique de la langue française, à l'oral. Les niveaux indiqués correspondent à l'échelle du Cadre Européen Commun de Référence pour les Langues (Conseil de l'Europe, 2001) à laquelle on peut se reporter.

Ces élèves ont connu une expérience antérieure de l'école, de quelques mois à un cursus relativement normal $(\mathrm{N})$ - dans ce dernier cas, l'élève était néanmoins illettrée - avec éventuellement un redoublement (R). Cinq ont connu des phases de déscolarisation (D). Tous ont été évalués en mathématiques, à leur arrivée en France. Ici, ce sont les résultats en calcul qui sont reportés : au mieux, l'élève maîtrise l'addition, la soustraction et la multiplication avec retenue (note équivalant à 3 points sur 5). Il arrive qu'aucune opération ne soit acquise. La compréhension écrite a été testée dans la langue de scolarisation connue, avec 3 textes possibles : niveau primaire (a), 6ème (b) ou fin de collège (c). Quatre élèves, en situation d'illettrisme, n'ont pas pu répondre aux questions posées sous forme de QCM.

Au vu de leurs compétences, ils ont été affectés dans des classes inférieures à leur classe d'âge et en fonction de la proximité géographique, ils ont pu suivre des cours en CLA ou en Cla-nsa.

\subsection{Leurs progressions linguistiques}

Ces 8 élèves sont arrivés la même année scolaire, à quelques semaines d'intervalle. Leur progression linguistique a été évaluée à partir de deux indicateurs. Le premier concerne les résultats au Delf, examen facultatif proposé en mai aux élèves récemment arrivés en France, qui évalue les compétences communicatives en compréhension et production écrites et orales. Le second indicateur consiste en un test linguistique, produit pour cette 
recherche et soumis lors de leur 3ème année à l'école française, entre janvier et mai 2011. Le test, d'une durée de 50 minutes, comprend un exercice à closure, une compréhension écrite extraite de Pisa (Programme International pour le Suivi des Acquis des élèves) proposée en 2000 (Bourny \& al., 2002 : 64-66) et une rédaction.

Les notes oscillent de 0 à 10 sur 20, c'est-à-dire d'un niveau A1.1 au niveau B1 en cours. Par ailleurs, ce même test a été proposé à 72 autres élèves qui avaient connu une scolarité régulière et qui étaient aussi scolarisés en France depuis 3 ans (Mendonça Dias, 2012). On constate ici qu'un élève se démarque : Ranko, qui réussit le Delf B1, certes de justesse et grâce aux compétences orales. Il rattrape un niveau similaire à celui d'élèves ayant suivi une scolarité régulière, contrairement aux autres NSA qui obtiennent les scores les plus faibles.

A titre indicatif, nous donnons un aperçu des compétences écrites maîtrisées et observées dans la rédaction dont le sujet est le suivant :

Sami est un nouvel élève. Il est arrivé en France le mois dernier. Il est dans ta classe.

Il veut déjà arrêter l'école car il ne comprend pas bien le français et il trouve l'école française trop difficile. À l'école, tu discutes avec Sami de sa décision.

Figure 3 - Les productions écrites des élèves NSA au test linguistique de l'année 3

\begin{tabular}{|c|c|}
\hline SHAMAN & $\begin{array}{l}\text { Je 'mdit sami pourquoi t'as arrté de l'ecole française parce qu t'es bensiea perend } \\
\text { lange de français il ma dit ci je vedrai bien prends lange français mes je benseroi } \\
\text { d'ordi }\end{array}$ \\
\hline DEMA & $\begin{array}{l}\text { salu sami } \\
\text { pour quoi sami tu ve arrete l'école } \\
\text { L'école ces emportan ça te donne françai } \\
\text { je c'est trop difficile faut premdre du courage }\end{array}$ \\
\hline CARINE & $\begin{array}{l}\text { Bonjour Sami c'est pour te dire que ne t'aquet pas tous sa va alle tu vas bien parle le } \\
\text { français. que tu ora beaucoup des amies. que moi aussi je ete come toi tu aura les } \\
\text { cours de soutin, en fle } 1 \text { au college que Dieu te protege tu conte beaucoup a on yeu } \\
\text { GROS BISOUS prend soi de toi A bienttot au revoir. } \\
\text { C'est CARINE [nom de famille] }\end{array}$ \\
\hline MINA & $\begin{array}{l}\text { Bonjour SAMI } \\
\text { Je suis Mina [nom de famille] j'ai } 15 \text { ans moi aussi j'étais comme toi je diais c'est } \\
\text { difficile mais je suis pas parti parce que je devais le faire pour et pas pour les autre. } \\
\text { Si tu veux vien avec moi comme sa ont travail ensemble Moi je te fais les cours de } \\
\text { français et toi les cours l'anglais parce que moi aussi je parle pas bien en l'Anglais, les } \\
\text { mercredi ont peu parti à paris pour visite les librerie. }\end{array}$ \\
\hline RANKO & $\begin{array}{l}\text { Bonjour Sami, tu est sur de bien vouloir partir? Je te demande ça parce que moi aussi } \\
\text { je ne savais pas de parler français je comprend ta situation mais, j'aimerai bien que } \\
\text { tu reste car si tu reste au collège tu vas apprendre de parler. Tu va aller apprendre la } \\
\text { langue en cours de FLS / FLS puis dans un an tu apprendras de parler, pas beaucoup } \\
\text { mais un peu. Après tu parllera avec tes copains puis tu parllera mieux. Voilà, je peux } \\
\text { rien faire pour toi d'après ce que je tes dit mais c'est par pour rien donc fait } \\
\text { attention ce que tu fait, réffléchi bien. Bon, à tout à l'heure Sami. }\end{array}$ \\
\hline
\end{tabular}


ABI, EVELYN et JUDITH ont rendu copie blanche pour l'exercice de production écrite car elles n'ont pas compris le sujet.

21 distinguent les bons travaux, dans l'ensemble des 80 copies récoltées des élèves récemment arrivés en France. Ici, ces critères sont validés pour Ranko et partiellement pour Mina et Carine. Les erreurs d'orthographe lexicale et grammaticale ne caractérisent pas les seules rédactions des élèves NSA mais elles sont plus récurrentes, gênant la lisibilité des textes. Les scripteurs rencontrent plus de difficultés à respecter l'orthographe phonétique, ce qui confirme la nécessité de travailler le rapport phonèmes/graphèmes de façon accrue. Les cours d'alphabétisation, qui distinguent la structure NSA, se justifient encore au cours de la 3ème année pour 6 élèves de cet échantillon.

On peut remarquer que les deux élèves les plus jeunes obtiennent les meilleurs résultats, ce qui n'est pas vérifié sur l'échantillon plus large où les élèves plus âgés progressent plus rapidement que leurs cadets.

\subsection{La vitesse d'apprentissage}

Nous avons déterminé le niveau linguistique atteint par des élèves NSA au cours de leur troisième année en France. Nous confrontons nos données avec les parcours des 43 élèves qui ont été inscrits dans une même Cla-nsa jusqu'à leur orientation au lycée, ce qui représente une inscription allant de quelques mois jusqu'à trois années passées, entre 2007 et 2011. Nous nous basons sur les comptes rendus établis par le Casnav pour connaître leurs profils linguistiques et scolaires à leur arrivée en France et nous estimons le niveau linguistique moyen atteint sur le critère de l'obtention du Delf. On constate qu'aucun des 3 élèves non scolarisés antérieurement et analphabètes à leur arrivée n'est en mesure de réussir le Delf A1 lors de sa 3ème année. En revanche, les élèves non francophones à leur arrivée en France, sachant déchiffrer dans leur langue d'origine, réussissent à effectuer des transferts : la lecture oralisée est assez rapide, mais ils ne comprennent pas le sens des textes, aussi ils passent le Delf A1 la 2ème, voire la 3ème année. Enfin, les élèves issus de pays francophones, peu scolarisés antérieurement ou dans des contextes difficiles, qui présentaient un niveau en lecture équivalant au cycle 2 acquis, peuvent être inscrits au Delf A1 quelques mois après leur arrivée. Seuls deux élèves francophones ont réussi de justesse le Delf $\mathrm{B} 1$ après trois années en France, grâce aux exercices de compréhension et production orales. La difficulté est accrue quand l'élève est non francophone et l'apprentissage est ralenti quand il ne connaît pas l'alphabet. Dans ce dernier cas, il ne peut pas encore prétendre au Delf A1 dans sa 3ème année pour réussir les épreuves écrites (cas de Abi).

24 Ainsi, la progression des élèves de la cohorte se révèle tout à fait normale au vu du rythme d'apprentissage confirmé par l'ensemble de cet échantillon, exception faite de Ranko dont la progression est supérieure à celle prévisible. Cet élève était inscrit en Cla ordinaire, sans cours spécifique (toutefois, dans notre échantillon, le faible nombre d'élèves n'ayant pas bénéficié de la Cla-nsa - 4 individus - ne nous permet pas d'en tirer de généralités). On constate que son frère aîné a un niveau moindre : très peu lecteur, 
échouant au Delf A1 la 2ème année, il est absent au moment du test et se déscolarise dans le courant de la 3ème année.

\subsection{Les progressions scolaires}

L'apprentissage est ralenti pour le public NSA. Nous avons vu que certains demeuraient encore peu lecteurs et peu scripteurs, même s'ils avaient été inscrits en Cla-nsa au moins une année scolaire. Est-ce que leurs compétences linguistiques risquent d'entraver la poursuite des études après le collège ? Les élèves de la cohorte qui ont quitté le collège ont été orientés vers des CAP (Certificat d'Aptitude Professionnelle) car le niveau atteint sur le plan communicatif ne leur permettait pas d'aspirer à des filières plus prestigieuses. Lors de la 3ème année, Bregan, très absentéiste, commence à se déscolariser. A la rentrée de leur 4ème année, deux autres élèves (Shaman et Evelyn) abandonnent les études pour des motifs personnels (maladie, dépression) et sont déscolarisés. Est-ce que les élèves NSA sont plus sujets à la déscolarisation en raison de leur niveau de compétences linguistiques?

Nous avons pu récolter les orientations des élèves de la Cla-nsa au cours des 5 dernières années. 43 élèves ont quitté le collège et voici les orientations (le nombre d'élèves figure entre parenthèses) :

Figure 4 - L'orientation de fin 3ème de 43 élèves issus d'une CLA-NSA au cours des années 2007 à 2011

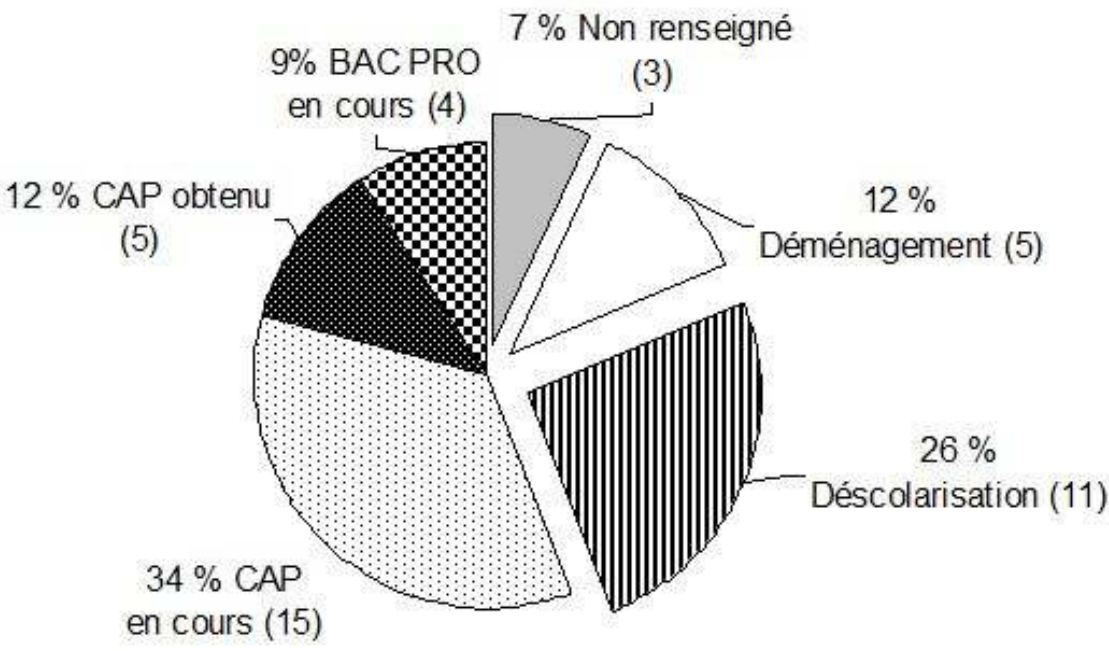

Un quart des élèves est déscolarisé. Hormis deux filles (Evelyn et Bénédicte, devenue maman), les élèves déscolarisés sont des garçons: ils cherchent à entrer dans la vie active. Ils étaient au niveau A2 à l'écrit et l'oral, au moment de leur orientation. Leurs compétences auraient pu laisser présager des parcours scolaires réussis mais quatre n'ont pas été s'inscrire au lycée, tandis que les cinq autres ont abandonné au fur et mesure de la première année de CAP. Étant donné les réussites d'autres élèves en CAP, avec des niveaux bien inférieurs (A1.1 à A1, pour plusieurs d'entre eux, à l'écrit), on peut conclure que les compétences langagières n'entravent pas la poursuite scolaire et l'obtention de diplôme professionnalisant, mais que c'est plutôt le rapport de l'élève avec l'école qui 
entraîne des déscolarisations au niveau lycée, quand bien même un accompagnement linguistique est proposé.

Nous avons pu récolter 16 bulletins scolaires au lycée. Les professeurs font état des "difficultés", termes récurrents à travers les appréciations, mais aussi de la bonne "volonté" et des "efforts", qui sont valorisés. On peut observer aussi le paradoxe pour ces élèves plurilingues d'obtenir souvent leur plus faible note en langue vivante, l'anglais, qu'ils ont débutée à leur arrivée en France. Le décalage d'âge par rapport à l'âge de référence caractérise la poursuite d'études en lycée et n'apparait pas ici comme un facteur de décrochage scolaire, contrairement aux données nationales ou sociologiques (Schiff, 2003).

\section{En conclusion}

Nous cherchions à déterminer des rythmes moyens concernant les progressions linguistiques et scolaires des élèves NSA, en prenant en compte leur profil et leur inscription éventuelle en Cla-nsa. Nous avons constaté que l'apprentissage nécessitait au minimum deux années scolaires pour qu'un élève NSA, au départ non francophone, atteigne les compétences écrites du niveau A2 en cours, qu'il soit ou non inscrit en Cla-nsa. Le niveau atteint n'entrave toutefois pas la poursuite des études en CAP et le décrochage scolaire apparaitt plutôt lié au rapport qu'entretient le jeune avec l'école et le monde professionnel. Suivant les académies, les inscriptions dans les dispositifs sont limitées souvent à une année. Cette étude a montré la nécessité de laisser du temps à l'apprentissage. Une meilleure de connaissance de ce public, à travers l'étude d'une cohorte plus large ou grâce à la mise en place d'un "groupe de réflexion, à l'échelon national", tel qu'il est proposé dans le rapport des Inspections Générales (Klein \& Sallé, 2009 : 173), permettrait de mieux comprendre les entraves à la formation diplômante et de favoriser la sortie scolaire avec une qualification.

\section{BIBLIOGRAPHIE}

Abdallah-Pretceille, M. \& Barbot, M.-J. (2003). « Le français comme langue étrangère et seconde ». In Collès, L., Dufays, J.-L. \& Maeder, C. (dir.). Enseigner le français, l'espagnol et l'italien - Les langues romanes à l'heure des compétences. De Boeck, coll. Savoir en pratique.

Bertrand, D., Viala, A. \& Vigner, G. (coord.) (2000). Le Français Langue Seconde. Paris : Centre National de Documentation Pédagogique, Collège-repères.

Bourny, G., Braxmeyer, N. Dupé, C, Rémond, M., Robin, I. et Rocher, T. (2002). Les compétences des élèves français à l'épreuve d'une évaluation internationale, Premiers résultats de l'enquête PISA 2000. Paris : Ministère de l'Éducation Nationale et de la Recherche, Direction de la Programmation et du Développement, Sous-direction de l'évaluation, pp. 64-66 et p. 93.

Caille, J.-P. \& Vallet L.-A. (1996). « Les élèves étrangers ou issus de l'immigration dans l'école et le collège français, une étude d'ensemble ", Les dossiers de l'Éducation et formations du MEN, $n^{\circ} 67$. 
Chomentowski, M. (2009). L'échec scolaire des enfants de migrants, L'illusion de l'égalité. Paris :

L'Harmattan.

Conseil de l'Europe (2001). Cadre européen commun de référence pour les langues : apprendre, enseigner, évaluer. Paris : Didier.

Circulaire $n^{\circ}$ 2002-100 du 25-04-2002. Organisation de la scolarité des élèves nouvellement arrivés en France sans maîtrise suffisante de la langue française ou des apprentissages. Bulletin Officiel spécial $\mathrm{n}$

- $10 \mathrm{du}$ 25-04-2002.

Circulaire $\mathrm{n}^{\circ}$ 2012-141 du 2-10-2012. Organisation de la scolarité des élèves allophones nouvellement arrivés. Bulletin Officiel $\mathrm{n}^{\circ} 37 \mathrm{du} 11-10-2012$.

Cuq, J.-P. (1991). Le Français Langue Seconde. Origines d'une notion et implications didactiques. Paris : Hachette.

Davin-Chnane, F. (2005). Didactique du FLS en France : le cas de la discipline "français" enseignée au collège. Villeneuve d'Ascq : Diffusion ANRT.

Francequin, G, (dir.) (1998). Kaleïdoscope polyphonique. Une boîte de réflexions et d'outils pour accueillir, connaître, scolariser et accompagner les élèves étrangers allophones au collège. Coménius, Conservatoire National des Arts et Métiers.

Haut Conseil à l'Intégration (2010). Les défis de l'intégration à l'école et Recommandations du Haut Conseil à l'intégration au Premier ministre relatives à l'expression religieuse dans les espaces publics de la République. Rapport au Premier ministre pour l'année 2010. Paris : Collection des rapports officiels.

Klein, C. \& Sallé, J. (2009). La scolarisation des élèves nouvellement arrivés en France. Paris : Ministère de l'Éducation Nationale, Ministère de l'Enseignement Supérieur et de la Recherche.

Lecoq, B. (coord.) (2012). Entrer dans la lecture, quand le français est langue seconde. Cap sur le Français de la scolarisation, Sceren.

Lorcerie, F. (1995). "Scolarisation des enfants d'immigrés : état des lieux et état des questions en France", Confluences-Méditerranée, $n^{\circ} 14$.

Ministère de l'Éducation Nationale, Ministère de l'enseignement supérieur et de la recherche (2009). Rapport annuel des Inspections Générales 2009. Chapitre 5 "La scolarisation des élèves nouvellement arrivés en France". Paris : La documentation française.

Mendonça Dias, C. (2012). Les progressions linguistiques des collégiens nouvellement arrivés en France. Villeneuve d'Ascq : Diffusion ANRT.

Millon-Fauré, K. (2011). Les répercussions des difficultés langagières des élèves sur l'activité mathématique en classe : le cas des élèves migrants. Thèse de doctorat, sous la direction d'A. MERCIER, Université Aix-Marseille I.

Rafoni, J.-C. (2008). Apprendre à lire en français langue seconde. Paris : L'Harmattan.

Schiff, C. (dir.) (2003). Non scolarisation, déscolarisation et scolarisation partielle des migrants. Programme interministériel de recherche sur les processus de déscolarisation. Paris : Ministère de l'Éducation Nationale.

Thomas, W. \& Collier, V. (2002). A National Study of School Effectiveness for Language Minority Students' Long-Term Academic Achievement. Final Reports, Center for Research on Education, Diversity and Excellence, UC Berkeley.

Verdelhan-Bourgade, M. (2002). Le français de scolarisation, pour une didactique réaliste. Paris : PUF. 
Vigner, G. (2002). « Quelle originalité pédagogique dans un enseignement du français, langue seconde ? ». In Martinez, P. (dir.), Besse, H., Cavalli, M., Cuq, J.-P. \& al., Le français langue seconde. Apprentissage et curriculum. Paris : Maisonneuve \& Larose.

\section{NOTES}

1. Depuis la circulaire de 2012, l'acronyme est désormais décliné en «Centre Académique pour la Scolarisation des enfants allophones Nouvellement Arrivés et des enfants issus de familles itinérantes et de Voyageurs ".

2. Dans cet article, nous conservons la terminologie en vigueur au moment de l'étude, à savoir Cla-nsa, mais les dispositifs viennent d'être renommés UPE2A (Unité Pédagogique pour Elèves Allophones Arrivants) depuis la circulaire de 2012.

3. Notre cohorte initiale était constituée de 17 élèves mais leur mobilité au sein de l'académie et hors académie n'a permis de suivre que 8 individus, en respectant tout le protocole de recherches.

4. Les dates de passation du test entre la fin janvier et mai 2011 dépendaient de la disponibilité des élèves (en stage ou en examen blanc) et des enseignants qui surveillaient l'épreuve.

5. L'échantillon initial compte 73 individus, mais seulement 43 d'entre eux ont une orientation après le collège, les autres étant encore au collège au moment de cette analyse.

6. Discours d'ouverture de monsieur Jack Lang, ministre de l'Éducation Nationale aux journées nationales d'étude et de réflexion sur la scolarisation des élèves nouvellement arrivés en France, 29 mai 2001, annexe 1 de la circulaire du 25 avril 2002 concernant la création des Casnav (circulaire $\left.n^{\circ} 2002-100,2002\right)$.

\section{RÉSUMÉS}

De façon inédite, nous abordons l'enseignement dispensé dans le système scolaire français à un public minoritaire et méconnu, mais ô combien spécifique: les collégiens primo-arrivants désignés "NSA", c'est-à-dire Non Scolarisés Antérieurement. A la problématique de l'apprentissage de la langue française, s'ajoute alors celle des compétences scolaires pour ces jeunes adolescents allophones, illettrés voire analphabètes, qui découvrent un cadre scolaire ainsi que de nouvelles références culturelles.

Nous cherchons à déterminer des rythmes moyens de progressions linguistiques, en fonction du cadre d'apprentissage et des facteurs individuels qui pondèrent les résultats, et nous mettons en perspective les niveaux linguistiques atteints avec les parcours scolaires. Dans notre étude, les évaluations initiales et les entretiens menés à l'arrivée de ces élèves font apparaître différents profils scolaires et linguistiques, remettant en question le sigle NSA, la majorité des élèves observés ayant, en fait, connu une courte scolarisation antérieure. Nous nous appuyons sur une cohorte de 8 élèves suivis 3 années, ainsi que sur les parcours, après le collège, de 43 élèves ayant été scolarisés dans un même dispositif entre 2007 et 2011. Pour connaître les caractéristiques des dispositifs, nous soumettons un questionnaire à 18 enseignants spécialisés. Après avoir étudié les résultats linguistiques et les orientations des apprenants, nous pouvons conclure que les élèves au départ non francophones mettent au moins deux ans pour atteindre le niveau A2 en cours, 
d'après le Cadre Européen Commun de Référence pour les Langues. Toutefois, des compétences écrites inférieures au A2 n'entravent pas la réussite d'un diplôme professionnalisant.

This study focuses on determining progress in French of migrant teenagers who come to France previously having never, or very little, attended school (Not at School Previously: NSA). Most of them are illiterate. They have to learn French, discover the educational and cultural context and develop new skills.

The aim of this study is to determine the average length of their progress in French while taking into account learning conditions and individual factors which might counterbalance the results. Progress in French is compared to the school record of each pupil. Eight pupils were followed over a period of 3 years and the records of 43 students registered in the type of same special class between 2007 and 2011 were studied. The results reveal that NSA pupils who didn't know French on arrival need at least 2 years to reach the A2 level (as defined by the Common European Framework of Reference for Languages). However, a pupil whose writing and reading skills are below the A2 level may still succeed in vocational training.

\section{INDEX}

Keywords : french as a second language, linguistic progress, Not at School Previously (NSA), illiteracy

Mots-clés : langue seconde, progression linguistique, CLA-NSA / UPE2A-NSA, élève, illettrisme

\section{AUTEUR}

\section{CATHERINE MENDONÇA DIAS}

Catherine Mendonça Dias est professeure de lettres modernes et formatrice au CASNAV de Bordeaux. Docteure en sciences du langage, rattachée à l'I3DL de Nice, qualifiée en sections 7 et 70 du CNU, elle fait partie de la SFERE (Structure Fédérative d'Études et de Recherches en Éducation) d'Aix-Marseille. Elle mène des recherches sur l'apprentissage et l'enseignement du français en tant que langue seconde en contexte scolaire.

Courriel : catherine.mendonca.dias@gmail.com

Toile : http://francaislangueseconde.awardspace.com/

Adresse: Collège Edouard Vaillant - Cours du Raccordement - 33000 Bordeaux 